\title{
Experiences and Lessons of Combating COVID-19 that Chinese Experts Shared with the World
}

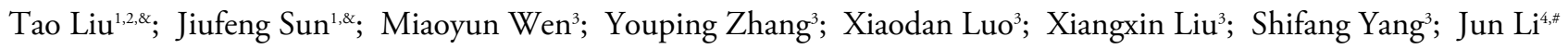

At the end of 2019, a novel viral pneumonia was detected in Wuhan City (1) and has spread rapidly throughout 215 countries, areas, and territories with more than 3.8 million cases and over 260,000 deaths as of May 8, 2020. This pneumonia, later named coronavirus disease 2019 (COVID-19), has become a global public security challenge. The World Health Organization (WHO) has declared it a pandemic and has called for the global community to work collaboratively to combat this novel pneumonia (2).

Since the early spread of COVID-19 in China, the Chinese government has implemented the most rigorous measures to prevent the spread of COVID-19 using the whole country's medical resources (3). Until the end of March 2020, the COVID-19 pandemic was essentially under control in China (3). Unfortunately, some other countries had insufficient experience in the ramping up and deployment of public health measures including prevention tactics, interventions, laboratory tests, clinical diagnosis, and treatment, which has resulted in continued suffering due to COVID-19. In order to collaborate with these countries to contain the global pandemic, the Chinese government decided to send expert teams to several countries, including Iran, Iraq, Italy, Serbia, Cambodia, Pakistan, Laos, Venezuela, Ethiopia, and Malaysia, to assist in the prevention of local COVID-19 transmission beginning at the end of February. The experiences and lessons shared by Chinese experts were intended to serve as references for these countries to inform policies on prevention and control. As one of the expert teams, here we summarized the major experiences and lessons that were shared with many countries still affected by COVID-19.

First, Chinese expert teams conducted field investigations and consulted with local governments, laboratory workers, health care workers, and citizens to collect information of the local situations of COVID19, laboratory testing capabilities, clinical treatment protocols, and control and prevention strategies and measures implemented. Then, they comprehensively assessed the situation and future trends of COVID-19 epidemic, which provided important information for them to communicate with local experts and to provide custom consultations for those countries.

Second, based on the questions proposed by local experts, the Chinese experts introduced major prevention and control strategies and measures implemented in China including "four early measures", "four priority measures", "four necessary measures", "three lines of defense", applications of "health quick response (QR) codes", and nonpharmaceutical intervention (NPI) measures (3). The "four early measures" includes early detection, reporting, isolation, and treatment of COVID-19 patients. The "four priority measures" includes concentrating patients, hospitals, experts, and treatment. The "four necessary measures" include all persons with need should be tested, admitted, treated, and isolated. The "three lines of defense" referred to joint travel quarantine at all travel stations including ports and wharfs, screening at hospitals, and management of focused population in communities. Applications of health QR codes involved monitoring and managing an individual's health status by having each individual apply for a health QR code after submitting their health status online. QR codes contained three categories: green, yellow, and red. Individuals with green status can move freely without registering to enter public facilities. Those with yellow codes were quarantined, and those with red codes were isolated for further laboratory testing or treatment. The NPI mainly included restricting inter-city movement for individuals, the identification and isolation of cases, and the reduction of inner-city travel and of contact to ensure social distancing (4).

Third, they shared clinical experiences on the diagnosis and treatment of COVID-19 patients. For example, they introduced the "Diagnosis and Treatment Protocol for Novel Coronavirus Pneumonia" in China (5). They also shared the usage of computed tomography (CT) scans for diagnosis of COVID-19 patients, selection of treatment drugs, treatment for pregnant women and newborns, zoning 
and classification management, optimization of medical resource allocation, multidisciplinary team building, determinants and predictors of poor prognosis, handling cases of surgery, and management of discharged patients.

Fourth, they introduced the roles of traditional Chinese medicine (TCM) in the treatment of COVID-19 patients. Medical doctors from TCM hospitals presented the effectiveness of using only TCM on clinic treatment of mild and moderate COVID-19 patients and combined protocols with western medicines on severe COVID-19 patients $(6-8)$. Some of them joined in the development of treatment guidelines for COVID-19 by using TCM in particular countries where TCM is popular, e.g. Malaysia.

Fifth, they introduced the worldwide developmental progress of the COVID-19 vaccine and the progress in China, the evidence of effectiveness of a potential vaccination, and the possible timeline of vaccine development. Particularly, they introduced two types of vaccines, a nucleic acid vaccine and an attenuated live vaccine, that were under phase II clinical trials in China. Ideally, both vaccines could be available at the end of 2020 or in early 2021. These communication activities provided state-of-the-art information and a great opportunity for future collaboration between China and other countries in the development of a COVID-19 vaccine.

Sixth, they shared the protocols of laboratory tests for the COVID-19 virus in China, such as methods involving specimen selection and collection and the sensitivity and specificity of detection methods. The virology experts also showed that temporal shedding of the virus in patients, particularly in discharged patients, provides important information for management of discharged patients. Moreover, they shared the sensitivity and specificity of rapid test kits, such as serological tests involving IgM, IgG, or IgA.

Seventh, they provided protocol for prevention of nosocomial infections, and protection of healthcare workers in China, such as the optimization of diagnosis and treatment procedures in hospitals, selection of personal protection equations (PPE) in different settings, management of PPE when experiencing shortages, disinfection protocol, and health management of healthcare workers.

Eighth, since COVID-19 was largely controlled by the end of February in China. Governments in lowrisk areas have resumed most economic activities have mostly reopened schools. Hence, Chinese experts shared experiences of the resumption and reopening. In particular, they presented the area-based risk rating strategy combined with usage of health QR codes. For example, all economic activities and normal life could be resumed in low-risk zones while focusing on the prevention of imported cases. By contrast, high-risk zones must prevent the export of cases and do not resume large-scale economic activities. Individuals with green QR codes do not need quarantine, while those with yellow or red code must follow the guidelines of quarantine or isolation for treatment.

Ninth, it has been demonstrated that the COVID19 epidemic may psychologically impact populations of COVID-19 survivors, children, the elderly, disabled people, patients with comorbidity diseases, family members of died patients, front-line medical workers, and quarantined persons (9-10). These psychological impacts are wide-ranging, substantial, and can be long lasting (11). Therefore, the Chinese experts shared the guidelines and practices for emergency psychological crisis intervention for COVID-19 patients or associated populations implemented in China (12).

Finally, the Chinese experts also conducted a series of online risk communication activities in countries where they provided support to share their knowledge with local citizens and overseas Chinese people including workers and students. They presented the general characteristics of COVID-19, its transmission routes, susceptibility of people, clinical symptoms, measures of prevention, and selection of PPE in different circumstances. This information improved their knowledge and ability to prevent COVID-19.

These experiences and lessons provided by the Chinese experts have significantly improved the understanding and ability to contain COVID-19 for experts in the supported countries. For example, some countries have developed a treatment protocol for COVID-19 using TCM, substantially enhanced their laboratory test capability for COVID-19 virus, developed an analogical health QR code system similar with China, and gradually resumed commercial activities and returned to routine work and living using an area-based risk rating strategy (13). Meanwhile, the Chinese experts also obtained experiences and learned lessons for containing COVID-19 from foreign experts in those supported countries. Overall, the overseas supporting activities implemented by the Chinese experts not only promoted potential research collaborations between China and these countries, but also substantially contributed to the containment of the COVID-19 pandemic worldwide. 
Fundings: This work is supported by the Ministry of Foreign Affairs of the People's Republic of China, the Health Commission of Guangdong Province, Guangdong Provincial Novel Coronavirus Scientific and Technological Project (2020111107001), and Science and Technology Program of Guangzhou (202008040004).

Acknowledgements: We thank all other expert team members who were sent to other countries. They did not receive compensation.

doi: $10.46234 / \mathrm{ccdcw} 2020.225$

\# Corresponding author: Jun Li, lijun1401@163.com.

\footnotetext{
Guangdong Provincial Institute of Public Health, Guangdong Provincial Center for Disease Control and Prevention, Guangzhou, China; ${ }^{2}$ School of Public Health, Southern Medical University, Guangzhou, China; ${ }^{3}$ Guangdong Provincial People's Hospital, Guangzhou, China; ${ }^{4}$ Guangdong Provincial Hospital of Traditional Chinese Medicine, Guangzhou, China.

\& Joint first authors.
}

Submitted: May 22, 2020; Accepted: July 20, 2020

\section{REFERENCES}

1. Li Q, Guan XH, Wu P, Wang XY, Zhou L, Tong YQ, et al. Early transmission dynamics in Wuhan, China, of novel coronavirus-infected pneumonia. N Engl J Med 2020;382(13):1199 - 207. http://dx.doi.org/ 10.1056/NEJMoa2001316.

2. World Health Organization. Coronavirus disease 2019 (COVID-19) Situation Report-52. https://www.who.int/emergencies/diseases/novelcoronavirus-2019/situation-reports/. [2020-05-9].

3. Editorial team of "China's Fight Against COVID-19". China's Fight Against COVID-19. China Daily, April 21, 2020. https://covid19.chinadaily.com.cn/a/202004/21/WS5e9e2c62a3105d50a3d17880.h tml. [2020-05-12].

4. Lu D. China is using mass surveillance tech to fight new coronavirus spread. New Scientist February 19, 2020. https://www.newscientist. com/article/mg24532703-600-china-is-using-mass-surveillance-tech-tofight-new-coronavirus-spread/. [2020-05-12].

5. National Health Commission of the People's Republic of China. Diagnosis and Treatment Protocol for COVID-19. http://www.nhc.gov. cn/xcs/zhengcwj/202001/808bbf75e5ce415aa19f74c78ddc653f.shtml. [2020-05-15]. (In Chinese).

6. Li QW, Wang H, Li XY, Zheng YJ, Wei Y, Zhang P, et al. The role played by traditional Chinese medicine in preventing and treating COVID-19 in China. Front Med 2020. http://dx.doi.org/10.1007/ s11684-020-0801-x.

7. Xu J, Zhang YF. Traditional Chinese medicine treatment of COVID19. Complement Ther Clin Pract 2020;39:101165. http://dx.doi.org/ 10.1016/j.ctcp.2020.101165

8. Luo ED, Zhang DY, Luo H, Liu BW, Zhao KM, Zhao YH, et al Treatment efficacy analysis of traditional Chinese medicine for novel coronavirus pneumonia (COVID-19): an empirical study from Wuhan, Hubei Province, China. Chin Med 2020;15:34. http://dx.doi.org/ 10.1186/s13020-020-00317-x.

9. Li LZ, Wang SH. Prevalence and predictors of general psychiatric disorders and loneliness during COVID-19 in the United Kingdom. Psychiat Res 2020;291:113267. http://dx.doi.org/10.1016/j.psychres. 2020.113267

10. Preti E, Di Mattei V, Perego G, Ferrari F, Mazzetti M, Taranto P, et al. The psychological impact of epidemic and pandemic outbreaks on healthcare workers: rapid review of the evidence. Curr Psychiat Rep 2020;22(8):43. http://dx.doi.org/10.1007/s11920-020-01166-z.

11. Duan L, Zhu G. Psychological interventions for people affected by the COVID-19 epidemic. Lancet Psychiatry 2020;7(4):300 - 2 . http://dx.doi.org/10.1016/S2215-0366(20)30073-0.

12. National Health Commission of China. A notice on the issuance of guidelines for emergency psychological crisis intervention in pneumonia for novel coronavirus infections. 2020. http://www.nhc.gov.cn/xcs/ zhengcwj/202001/6adc08b966594253b2b791be5c3b9467.shtml. [2020-05-12]. (In Chinese).

13. Bernama. Traditional meds used for 95pct of COVID-19 cases in Hubei-China team. Apr 27, 2020. https://www.malaysiakini.com/news/ 522767. [2020-07-14] 